\title{
Stochastic processes govern invasion success in microbial communities when the invader is phylogenetically close to resident bacteria
}

\author{
Marta Kinnunen $\mathbb{D}^{1,2} \cdot$ Arnaud Dechesne $\mathbb{D}^{1} \cdot$ Hans-Jørgen Albrechtsen $\mathbb{D}^{1} \cdot$ Barth F. Smets $\mathbb{D}^{1}$
}

Received: 18 December 2017 / Revised: 12 April 2018 / Accepted: 11 May 2018 / Published online: 12 July 2018

(c) International Society for Microbial Ecology 2018

\begin{abstract}
Despite recent efforts in identifying the determinants of invasion in microbial communities, experimental observations across different ecosystems are inconclusive. While relationships between resident community diversity and invasion success are often noted, community diversity says little about community assembly processes. Community assembly processes may provide a more inclusive framework to explain—and potentially prevent or facilitate—invasion. Here we let replicate nitrite-oxidizing bacterial guilds assemble under different conditions from a natural source community and study their compositional patterns to infer the relative importance of the assembly processes. Then, an invader strain from that same guild was introduced at one of three propagule pressures. We found no significant correlation between community diversity and invasion success. Instead, we observed that the effect of selection on invasion success was surpassed by the effect of drift, as inferred from the substantial influence of propagule pressure on invasion success. This dominance of drift can probably be generalized to other invasion cases with high phylogenetic similarity between invader and resident community members. In these situations, our results suggest that attempting to modulate the invasibility of a community by altering its diversity is futile because stochastic processes determine the invasion outcome. Increasing or reducing propagule pressure is then deemed the most efficient avenue to enhance or limit invasion success.
\end{abstract}

\section{Introduction}

Biological invasions can impact resident communities and ecosystems by facilitating fluctuations in biodiversity and in this way alter community function and productivity. For macro-organisms, many factors enabling successful invasions have been identified and considerable scientific effort has been devoted to elucidate the determinants of invasion in microbial communities in order to prevent or promote the establishment of new community members [1-3].

Electronic supplementary material The online version of this article (https://doi.org/10.1038/s41396-018-0202-1) contains supplementary material, which is available to authorized users.

Barth F. Smets

bfsm@env.dtu.dk

1 Department of Environmental Engineering, Technical University of Denmark, Bygningstorvet 115, 2800 Kgs, Lyngby, Denmark

2 Present address: Novozymes A/S, Microbial Discovery, Krogshoejvej 36, Bagsvaerd DK-2880, Denmark
Competition with resident community members has primarily been suggested to determine invasion success, and strong competition decreases invasion success [4-6]. The level of competition is usually inferred from resident community diversity [7] or from the phylogenetic distance between the invader and resident community members [8]. It is suggested that with small phylogenetic distance between invader and resident community members, resident community members impose strong competition on the invader type because phylogenetic similarity implies ecological similarity [8], which would reduce invasion success [9-12]. In a similar vein, it has been theoretically [1] and experimentally [13-15] suggested that biologically diverse communities are more resistant towards invasion, as originally proposed by Elton [7]. The most commonly cited reason is that more diverse communities are able to utilize resources more efficiently, thus leaving little resource space for invaders, and have higher probability of hosting a type capable of out-competing an invader. However, when community diversity is examined as a single factor determining invasion success without considering the 
specific context for interpretation, false conclusions are likely [16] because other community assembly processes (i.e., selection, drift, dispersal or speciation) contributing to resident community diversity are often neglected [17].

Competition between invader and resident community members is mainly investigated using synthetically assembled microbial communities [13, 18] with limited similarity to natural communities. Synthetically assembled microbial communities allow testing invasion success at different (controlled) diversity levels as well as carefully chosen phylogenetic distances between community members. However, this approach does not allow testing how all community assembly processes affect invasion because oftentimes only one or two processes (selection and/or drift) govern community assembly when establishing synthetic communities with no history of interaction. It is thus unclear if resident community diversity and phylogenetic distance between invader and resident community members can serve as general predictors of invasion success, beyond synthetic communities.

On the other hand, recent studies have suggested that microbial community assembly is more stochastic [19] than recognized in the studies focusing on competition, and that invasion success would primarily depend on propagule pressure $[20,21]$ (the relative abundance of the invader to the resident community), as postulated for communities of macro-organisms [22-24]. While resident community diversity has predicted invasion in several cases $[13,15,21]$, a similar amount of evidence supports propagule pressure as determinant of invasion [20, 21]. The lack of consensus across studies may be because the investigations are often limited to only one determinant of invasion. For example, sometimes communities with different diversities are subject to invasion at single propagule pressure $[9,13,15,25,26]$, or the phylogenetic distance between resident community members and invader is so large that it is highly improbable that it accurately represents competition for an ecological niche [14].

Hence, here we subject guilds of nitrite-oxidizing bacteria (NOB) to invasion by a NOB strain and thus investigate invasion outcome in communities where competition is expected, and where phylogenetic distance between invader and resident community members is low. We hypothesize that with low phylogenetic distance to the resident community members, invasion success is influenced by propagule pressure. Since low phylogenetic distance can confer ecological similarity, neither the resident community members nor the invader would have a competitive advantage and the effect of drift would govern invasion success.

\section{Materials and methods}

\section{Invader cultivation}

A culture of Nitrotoga HW29 was used as the invader, grown according to its enrichment conditions [27] in 250-mL cell-culture flasks over a 3-month period. After 3 months, NOB mineral medium was replaced with sterilized non-chlorinated tap water for one month to adjust the invader to the conditions of the resident community. No changes in nitrite removal dynamics were observed in response to this change in the medium. Before the onset of the invasion, all batch cultures were combined and the cell density was determined using a Thoma cell-counting chamber. Then, dilutions of the culture in tap water were spiked with either $0.3 \mathrm{mM}$ or $0.03 \mathrm{mM}$ nitrite to introduce the invader to resident communities with high and low nitrite loading, respectively.

\section{Invasion in flow-through microcosms}

The experimental set-up consisted of 40 parallel flowthrough microcosms. Biofilms developed on Filtralite NC 0.8-1.6 filter material (Saint-Gobain Byggevarer A/S, Oslo) fed with tap water spiked with nitrite at a constant flow rate of $0.43 \mathrm{~L}$ per day under ambient temperatures $\left(23-25^{\circ} \mathrm{C}\right)$. One set of 20 replicates was fed with tap water with $0.3 \mathrm{mM}$ $\mathrm{NO}_{2}{ }^{-}-\mathrm{N}$ addition, while another set of 20 replicates received 10-fold lower nitrogen concentration, $0.03 \mathrm{mM}$ $\mathrm{NO}_{2}{ }^{-}-\mathrm{N}$. Resident community biofilms were allowed to develop for 60 days, after which 4 random columns were destructively sampled and used as before invasion reference (called 'initial' in results and discussion) and as inocula for batch microcosms (see below), while the remaining columns were subjected to invasion. Three different propagule pressures were applied; such as the total invader cells after a $24 \mathrm{~h}$ of continuous invasion were estimated to represent on average 1,10 and $100 \%$ of resident NOB cells. The absolute abundance of the resident NOB cells before invasion was estimated from the nitrite removal dynamics and average NOB growth kinetics according to Rittmann and McCarty [28]. We observed complete nitrite removal from day 30 onwards, resulting in a total of $0.4 \mathrm{mg} \mathrm{NO}_{2}{ }^{-}-\mathrm{N}$ consumed by the resident bacteria at low nitrite loading, and $4 \mathrm{mg} \mathrm{NO}_{2}{ }^{-}-\mathrm{N}$ at high nitrite loading, yielding $\sim 10^{7}$ and $10^{8}$ cells per microcosm at low and high nitrite loading, respectively. Each propagule pressure treatment consisted of 4 replicates, whereas 4 replicates at both nitrogenloadings were maintained as controls without invader (referred to as 'none'). The flow-through microcosms were operated for another 14 days following the invasion after which all material was harvested ('final after invasion' - or 'final') and DNA extracted. 


\section{Invasion in batch microcosms}

Batch microcosms were established in $250-\mathrm{mL}$ cell-culture flasks with the same nitrite concentrations as in the flowthrough microcosms. Nitrite-spiked sterile tap water was used as medium and $0.5 \mathrm{~g}$ of wet filter material from the initial community from either high or low nitrite loading flow-through columns was added as inoculum. The flasks were subject to rigorous shaking to detach the cells from the filter material and promote growth in suspension. We assumed the absolute abundance of inoculated resident NOB cells to correspond to the abundance estimated for the flowthrough microcosms, but corrected for filter material used for inoculation $\left(10^{3}\right.$ cells $\mathrm{ml}^{-1}$ and $10^{4}$ cells $\mathrm{ml}^{-1}$ in low and high nitrite loading batch microcosms, respectively). We used this to determine the correct propagule pressure with similar ratios as in the flow-through microcosms: 1,10 and $100 \%$ of average resident NOB cells. In batch microcosms, the invader cells were added at the same time as the inoculum filter material. The absolute abundance of resident NOB cells differed in flow-through and batch microcosms. Therefore, our experiments included 6 different conditions of absolute propagule pressure, and 3 of relative propagule pressure.

The nitrite removal was measured regularly and when depleted, half of the medium was replaced. After 5 transfer events, the cells were recovered by filtering the total microcosm volume and the retentate was subjected to DNA extraction.

\section{DNA extraction}

DNA from was isolated using the FastDNA ${ }^{\mathrm{TM}}$ SPIN Kit for Soil and the FastPrep ${ }^{\circledR}$ Instrument (MP Biomedicals, Santa Ana, CA) according to the manufacturer's instruction at room temperature. DNA from liquid microcosms (batch and invader culture) was isolated after filtering $(100 \mathrm{~mL}$ of invader cell culture and all $250 \mathrm{~mL}$ of the batch microcosms) through sterile 0.1 um filters using DNeasy ${ }^{\circledR}$ PowerWater $^{\circledR}$ kit (QIAGEN, Hilden, Germany) according to manufacturer's instructions at room temperature. The concentration and purity of extracted DNA were checked using NanoDrop ${ }^{\mathrm{TM}} 2000$ Spectrophotometer (Thermo Fisher Scientific, Wilmington, DE). DNA was then stored at $-20^{\circ} \mathrm{C}$ for subsequent molecular analyses.

\section{qPCR}

Real-time qPCR assays were performed with a Roche LightCycler $^{\oplus} 96$ Instrument (Basil, Switzerland). Reaction mixtures $(25 \mu \mathrm{l})$ contained $12.5 \mu \mathrm{l} \mathrm{SYBR}^{\circledR}$ Green qPCR Mastermix (iQ ${ }^{\mathrm{TM}} \mathrm{SYBR}^{\circledR}$ Green Supermix; Bio-Rad, Hercules, CA) $1 \mu \mathrm{l}$ forward and reverse primers $(20 \mu \mathrm{M}), 5 \mu \mathrm{l}$ of template DNA (adjusted to $2 \mathrm{ng}$ DNA $\mu \mathrm{l}^{-1}$ ) and $5.5 \mu \mathrm{l}$
PCR-grade water. Total bacteria were quantified based on 16S rRNA gene copy numbers using the Eubacterial primer set $1055 \mathrm{f}-1392 \mathrm{r}$ as described in Terada et al., [29]. On average 2.5 copies of $16 \mathrm{~S}$ rRNA gene was estimated per cell, according to $r r n \mathrm{DB}$ [30], with the assumption, based on previous work [31], that majority of the community belongs to Gallionellacea and Nitrospiraceae. Nitrospira cells were quantified using Nitrospira-specific qPCR with primer set NTS232f [32] and Nsr1264r [33] targeting the $16 \mathrm{~S}$ rRNA genes. Cell numbers were calculated assuming a single $16 \mathrm{~S}$ operon per cell $(\mathrm{rrn \textrm {DB }})$.

The primer set Ntoga118F (5'-CTTTCAGCCGGAAAGAAAACGCA-3') and Ntoga840R (5'-CTAAGGAAGTCTCCTCCC-3') was developed for this study to target the 16S rRNA gene of Nitrotoga cells. The primers were designed based on Nitrotoga amplicon sequences retrieved from a previous experiment where Nitrotoga was enriched from tap water spiked with nitrite [31]. The designed primers cover $27 \%$ of known Nitrotoga in the SILVA rRNA database (including that of Nitrotoga HW29) and $100 \%$ of the tap water enriched Nitrotoga from previous experiment [31]. These primers target a $175 \mathrm{bp}$ product that was verified by constructing a clone library of 180 clones, all of which were determined to belong to Nitrotoga genus. Amplification consisted of 35 cycles of $94^{\circ} \mathrm{C}$ for $30 \mathrm{~s} ; 63^{\circ} \mathrm{C}$ for $30 \mathrm{~s} ; 72^{\circ} \mathrm{C}$ for $60 \mathrm{~s}$, followed by melting curve analysis.

\section{Sequencing and amplicon library}

Extracted DNA from all samples was PCR-amplified using primer set PRK341F (5'-CCTAYGGGRBGCASCAG-3') and PRK806R (5'-GGACTACNNGGGTATCTAAT-3') for 35 cycles, to amplify the V3-V4 hypervariable regions [34]. Purified PCR products were sequenced on the Illumina MiSeq platform at the DTU Multi Assay Core Center (Lyngby, DK).

All raw 16S rRNA gene amplicons were processed following the DADA2 (version 1.0.3) pipeline with default settings [35]. These sequence variants were classified based on the SILVA prokaryotic reference database version 123 . Invader sequence was identified based on the $100 \%$ similarity to $16 \mathrm{~S}$ sequence of HW29 found in NCBI database by constructing a phylogenetic tree of all Nitrotoga sequence variants and including the Nitrotoga HW29 sequence as a reference (Supplementary Figure S5). All sequences have been submitted to NCBI Sequence Read Archive under accession number SRP116646.

\section{Statistical analysis}

All statistical tests were performed in $\mathrm{R}$. The relative abundance of invader sequence variant of all NOB as well 
as the similarity between biological replicates were determined using phyloseq package [36]. Phylogenetic distances and Bray-Curtis distances were calculated and plotted as NMDS using phyloseq package. Phylogenetic diversity was calculated using PhyloMeasures package [37]. The statistical difference of the phylogenetic diversity between treatments was determined using a Wilcoxon signed-rank test, comparing the non-invaded control groups at different nitrite loadings. The absolute cell numbers obtained by qPCR were compared using two-way ANOVA tests, with factors corresponding to nitrite loading rate and propagule pressure. Correlations between descriptive indices and invader relative abundance were determined using linear regression model and the significance of the difference in correlation between treatments was determined using twoway ANOVA test.

\section{Results and discussion}

\section{Ecological processes governing resident community assembly}

We enriched 40 resident communities from tap water source community in flow-through microcosms subjected to two nitrite-loading regimes to support the coexistence of competing NOB genera [31]. We described the resident community composition after 60 days of operation (further referred to as initial community) and 14 days after the invasion event (referred to as final community). The flowthrough microcosms are expected to facilitate selection, drift and dispersal. We also inoculated batch microcosms with the initial community from the flow-through microcosms to establish a set of microcosms where community assembly processes were simplified by elimination of dispersal. The composition of the batch microcosms was characterized after the inoculation of the 'resident' community together with the invader, representing the starting community after the inoculation (initial community), and after five subsequent transfer events (final community). While adding invader simultaneously with the resident community can be viewed as co-assembly, and not invasion, here, we emphasize that the inoculum material originating from the flow-through microcosms already had 60 days of co-evolution and therefore can be considered as resident community, even when introduced at the same time with the invader cells.

Faith's phylogenetic diversity of the resident NOB guild was significantly lower at high vs low nitrite loading (Table 1) in the flow-through microcosms (Wilcoxon test $p=0.02$ ) but not in batch microcosms (Wilcoxon test $p=0.15)$. This low phylogenetic diversity in flow-through microcosms corresponded to resident communities, where Nitrotoga dominated over Nitrospira at high nitrite loading, and was consistent with known differences in nitrite affinity and specific growth rates of these two genera [38].

Table 1 Effect of community assembly processes and propagule pressure on frequency of invader establishment

\begin{tabular}{|c|c|c|c|c|c|c|c|}
\hline & \multicolumn{2}{|c|}{ Flow-through microcosms } & \multicolumn{5}{|c|}{ Batch microcosms } \\
\hline & Low nitrite loading & $\begin{array}{l}\text { High nitrite } \\
\text { loading }\end{array}$ & Low nitr & ite loading & & High nitrite & loading \\
\hline SELECTION (direction) & $\begin{array}{l}+(\text { Nitrotogal } \\
\text { Nitrospira })\end{array}$ & $+++($ Nitrotoga $)$ & $++(N i t)$ & ospira) & & $++($ Nitros $)$ & ira) \\
\hline DRIFT & ++ & + & ++++ & & & +++ & \\
\hline Selection to drift ratio ${ }^{\mathrm{a}}$ & ++ & ++++ & + & & & + & \\
\hline DISPERSAL & ++ & + & 0 & & & 0 & \\
\hline Faith's phylogenetic diversity ${ }^{b}$ & $0.38 \pm 0.05$ & $0.31 \pm 0.006$ & $0.39 \pm 0$ & & & $0.34 \pm 0.03$ & \\
\hline Mean phylogenetic distance from invader ${ }^{c}$ & $0.02 \pm 0.002$ & $0.009 \pm 0.004$ & $0.07 \pm 0$. & & & $0.08 \pm 0.04$ & \\
\hline Nearest phylogenetic distance from invader ${ }^{\mathrm{d}}$ & 0.011 & 0.011 & 0.011 & & & 0.011 & \\
\hline Relative propagule pressure & $+\quad++\quad++++$ & $+\quad++\quad+++$ & + & ++ & ++++ & $+\quad++$ & +++ \\
\hline Invader establishment frequency ${ }^{\mathrm{e}}$ & $\begin{array}{lll}0 & 0 & 1\end{array}$ & $\begin{array}{lll}0 & 0 & 0.5\end{array}$ & 0.5 & 0.5 & 1 & $\begin{array}{ll}0 & 0.5\end{array}$ & 0.5 \\
\hline Rel. abundance of invader $(\%)^{\mathrm{f}}$ & $13.9 \pm 7.6$ & $8.1 \pm 3.2$ & $16.4 \pm 0$ & $10.1 \pm 0$ & $24.5 \pm 23.7$ & $0.9 \pm 0$ & $9.8 \pm 0$ \\
\hline
\end{tabular}


As pointed out above, diversity indices without context do not say much regarding the ecological processes shaping the resident NOB guilds. Therefore, in this study, we elaborated on the relative contribution of the four processes (i.e. selection, drift, dispersal and speciation) that govern community assembly [39], and subsequently determine invasion outcome.

In Table 1, we provide an overview of the evaluation of the importance of selection, drift and dispersal in the resident communities. We can neglect speciation, as it is unlikely in the short timeframe of the experiment that new types arise and achieve significant abundance. Our interpretation of the strength of processes acting on the resident communities is based on the dynamics and consistency across replicates of the composition of the non-invaded control communities (Fig. 1, Supplementary Figures S1 and S2) and a conceptual synthesis of community ecology [39]. We measure stochastic effects as within-group distances of replicate communities, such that large dissimilarities represent strong effect of stochastic community assembly processes. Similarly, small dissimilarities between replicate communities point towards strong effect of selection, as suggested in Evans et al. [40].

Dispersal was relevant only in flow-through microcosms since they were open to the environment, in contrast to the batch microcosms, which were fed sterile tap water spiked with nitrite. Dispersal can influence the diversity, composition, as well as functioning of a community and the effect of dispersal seems to be enhanced in smaller communities [44]. For NOB guilds newly assembled from tap water, the contribution of dispersal is low, compared to the contribution of selection and drift [31]. Hence, we focus on the relative importance of selection and drift from here on.

The similarity between the resident communities independently assembled from the same source community was highest in resident communities assembled under flowthrough conditions (Fig. 2), which indicates that selection was most important. The direction of selection was affected by the nitrite loading, as seen from the difference in the ratio of Nitrotoga to Nitrospira at different nitrite loadings. At the time of invasion, Nitrospira abundance had not reached steady state (Fig. 1) since it increased significantly during the 14 days after the invasion event as seen by comparing the 'initial resident' and 'final' community fractions (ANOVA low nitrite $p=0.01$; high nitrite $p=0.05$ ). In low nitrite loading flow-through microcosms, selection pressure was positive towards Nitrospira, as Nitrospira increased in abundance relative to Nitrotoga. Even though Nitrospira also increased significantly in abundance in high loading flow-through microcosms, the Nitrotoga-to-Nitrospira ratio was higher than in the low nitrite loading, indicating strongest selection towards resident Nitrotoga. While one Nitrotoga type has been found to be one of the key
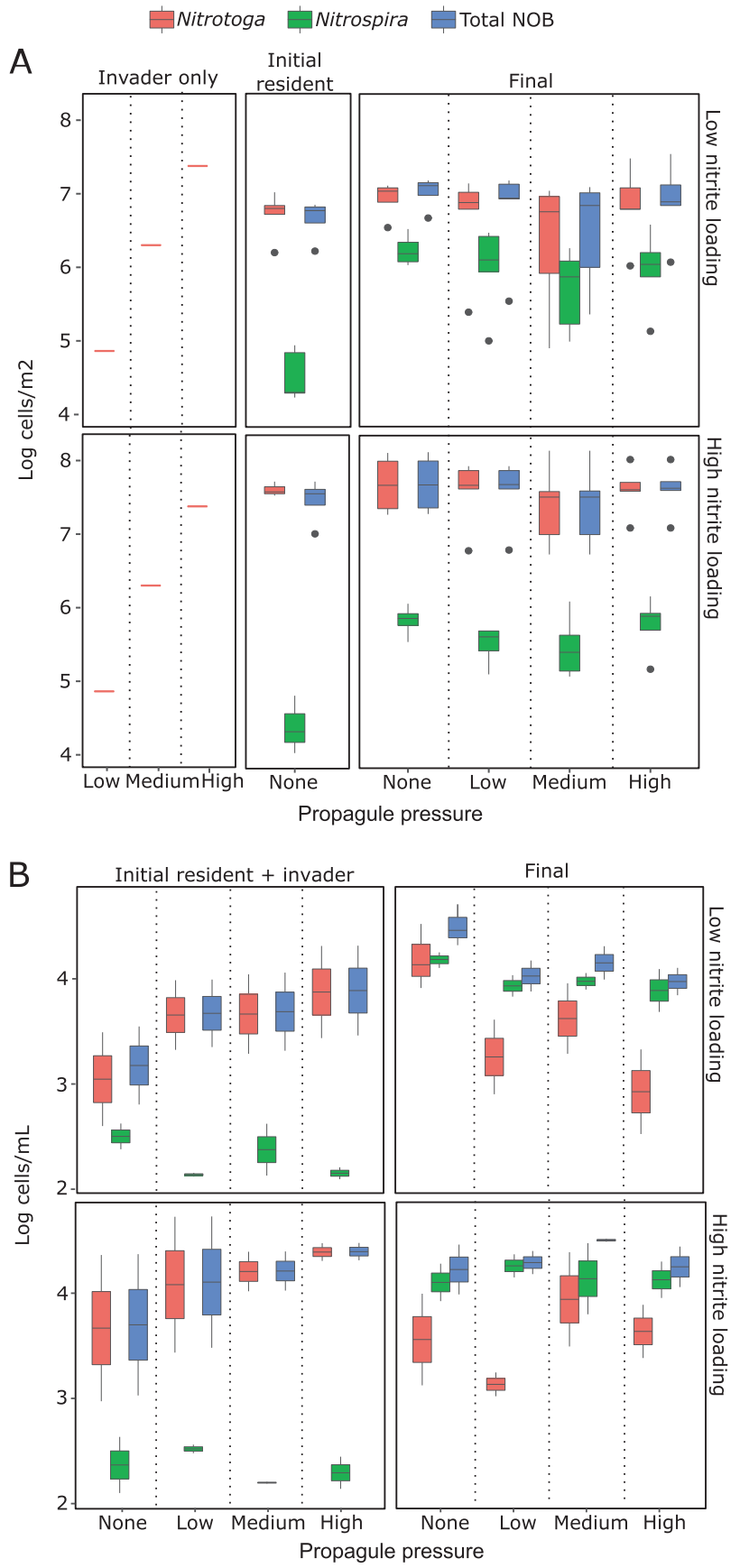

Fig. 1 Box-and-whisker plot representing the density of NOB in (a) flow-through and (b) batch microcosms before (initial) and after invasion (final) determined by targeted qPCR. The initial community composition was measured before invasion for flow-through microcosms and after first transfer for batch microcosms. Propagule pressure none refers to the non-invaded control microcosms operated in parallel with invaded microcosms

nitrite-oxidizers in wastewater treatment [42], indicating its adaptability at higher nitrite concentrations, little is known about the nitrite affinity of different Nitrotoga strains in drinking water communities. Previous studies on competition between Nitrospira and Nitrotoga in drinking water 


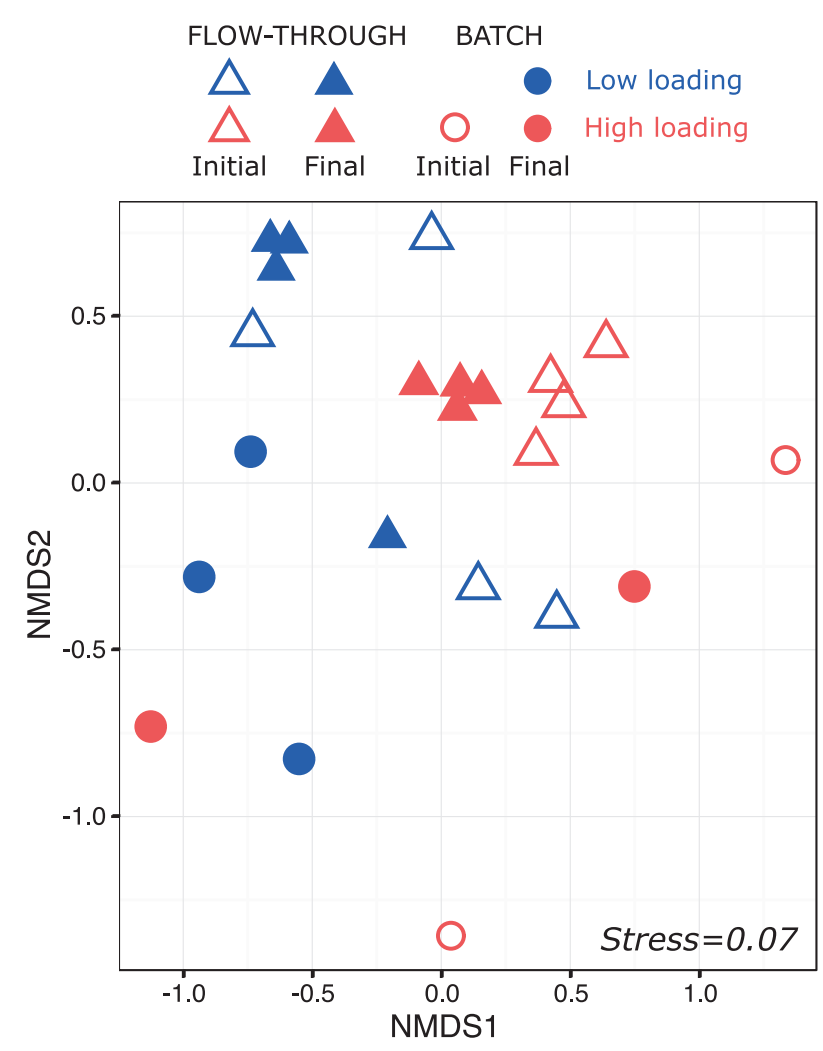

Fig. 2 Similarities between non-invaded resident communities independently assembled from the same source community in flowthrough and batch microcosms under two nitrite loading, based on nonmetric multidimensional scaling ordinations of Bray-Curtis distances across community structures inferred from total community $16 \mathrm{~S}$ rRNA amplicon libraries

treatment, however, have also observed the dominance of Nitrospira at low nitrite loading conditions that is outcompeted by Nitrotoga at higher nitrite loading conditions $[31,43]$.

Interestingly, the selection in the batch microcosms favored Nitrospira under both loading conditions (see final community on Fig. 1 and Supplementary Figure S2). This can be due to the dynamic nitrite-loading in these microcosms, causing nitrite concentration changes over time, providing niches for NOB with a range of affinities for nitrite. In flow-through microcosms, the nitrite concentration attains steady-state (Supplementary Figure S1), likely selecting for NOB with a narrower range in substrate affinity. On the basis of this, we expect the invader Nitrotoga strain to be less competitive at low nitrite loading than high nitrite loading, in resident communities dominated by competition.

Next, we estimated the relative contribution of drift to the assembly of the resident communities. In both flow-through and batch microcosms, significantly lower guild abundance was observed at low nitrite than at high nitrite loading, as expected (ANOVA flow-through $p<0.0001$; batch $p=0.04)$. Communities with low abundance are theoretically more affected by drift than communities with more members [44]. The higher dissimilarities between replicate communities after 60 days of low vs. high nitrite loading also support this (Fig. 2). The contribution of selection over drift was inferred to be highest in high nitrite loading flowthrough microcosms based on the high similarity in composition of communities independently assembled from the same source community (Fig. 2). In contrast, the contribution of selection over drift was inferred to be lowest in batch microcosms (Fig. 2). In these microcosms, half of the community was regularly removed, promoting higher turnover in replacement of removed community members and amplifying the effect of drift compared to the flowthrough system.

We can now explain the underlying causes for the differences in the observed phylogenetic diversity of resident NOB guilds (Table 1) based on the community assembly processes discussed above: we saw no significant difference in NOB phylogenetic diversity in batch microcosms, supporting our interpretation that similar processes dominate the community assembly in batch microcosms irrespective of the nitrite loading regime. In flow-through microcosms, however, the influence of selection over drift varied between the two nitrite loading treatments: in the high nitrite loading microcosms higher selection to drift ratio resulted in significantly lower phylogenetic diversity (Wilcoxon test $p=0.02$ ) and the dominance of few community members with high relative fitness.

\section{Successful establishment of the invader}

In the flow-through microscosms, the resident NOB guild was subject to continuous invasion during a $24 \mathrm{~h}$ period by a culture of Nitrotoga HW29 [27], while the invader was introduced simultaneously with the resident community in the batches. In flow-through microcosms, the invader strain was subjected to competition with 2 other Nitrotoga and 6 Nitrospira types at low loading conditions and 3 Nitrotoga and 3 Nitrospira types at high loading conditions (Supplementary Figure S2). We used three defined concentrations of invader cells (see invader qPCR data on Fig. 1) to achieve low, medium and high relative propagule pressure conditions (estimated to be equivalent to 1,10 and $100 \%$ of the total resident NOB population), with the aim to test the effect of drift on invasion success. Following the introduction of the invader strain, we allowed another five biomass turnover times (approximately 14 days, estimated from the resident community cell numbers and nitrite loading rates) before sampling the follow-through microcosms. This time the establishment ensured that, if observed, invader persistence would indicate an active population rather than residual invader cells.

Figure 1 shows that the invader cell addition did not significantly change the total NOB cell numbers after 
5 biomass turnover times, except in the low nitrite loading batch microcosms (Wilcoxon test $p=0.02$ ). The resident community displayed complete nitrite removal during 30 days before the invasion event (Supplementary Figure S1), suggesting that the resident community had reached its carrying capacity by the time of the invasion event. Hence, if established, the invader Nitrotoga displaced some of the resident NOB types or established at low relative abundance.

Based on amplicon sequencing, we could monitor the establishment of the invader strain-as its sequence was not present in the original resident community. The invader Nitrotoga strain was only established in the flow-through microcosms at high propagule pressure, whereas in batch microcosms, it was established at almost all propagule pressures, although at different relative abundance (Table 1). The frequency of establishment increased in both batch and flow-through microcosms with increasing propagule pressure (Fig. 3).

\section{Descriptive indices of community composition fail to predict invasion success}

First, we tested the diversity-invasibility hypothesis in NOB guilds. We determined correlations between the relative abundance of invader (relative to total NOB) and the phylogenetic diversity of the resident community (Supplementary Figure S6) as well as invader relative abundance and nearest (Supplementary Figure S7) and mean phylogenetic distance to the resident community members (Supplementary Figure S8). We need to emphasize that comparing the guild diversity and phylogenetic distance between invader and resident community is only appropriate for replicate microcosms assembled by similar processes because different assembly processes contribute differently to community diversity as well as invasion

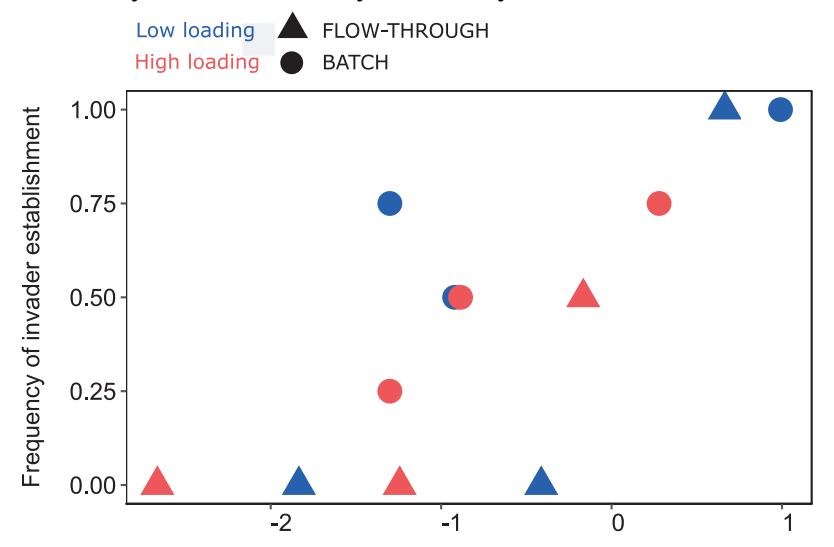

Log ratio of invader to resident NOB

Fig. 3 Frequency of invader establishment in 4 replicate microcosms at different propagule pressure (ratio of invader to resident NOB) and in different experimental microcosms (circles for batch microcosms and triangles for flow-through microcosms) success. In flow-through microcosms, the communities assembled at high and low nitrite loading were governed by different processes; hence, combining the replicates from different treatments would encourage false conclusions of what governs invasion success. Hence, we determined correlations separately for invasion in communities from high nitrite loading and low nitrite loading flow-through microcosms. Because we inferred no difference in dominating assembly process in batch microcosms, we combined the replicate communities at different nitrite loading regimes. We reject the common hypothesis that invader establishment is negatively correlated with resident community diversity (Supplementary Figure S6). While we observed a negative trend between the resident community diversity and the relative abundance of the invader after establishment in flow-through microcosms, we failed to observe any significant correlation in flow-through microcosms, contrary to batch microcosms. We observed a significant positive correlation between resident community diversity and the relative abundance of the invader in batch microcosms. Clearly, phylogenetic diversity of the resident community is not a universal predictor for invasion resistance in a functional guild.

Another metric used to predict invasion success-the nearest and/or average phylogenetic distance to the resident community [45] —is assumed to be positively correlated with invasion. However, because selection acts similarly on community members that are phylogenetically similar [8], we hypothesized here that drift would, therefore, determine invasion success when phylogenetic distance between invader and the resident community is low. We neither saw significant correlation between the nearest (Supplementary Figure S7) nor the average phylogenetic distance and relative abundance of the invader (Supplementary Figure S8). Our observations indicate that, when the relative importance of selection over drift in communities is low, mean phylogenetic distance to the resident community correlates negatively with invader relative abundance. Failing to see consistent correlations between invasion success and resident community diversity as well as phylogenetic distance between invader and resident community members, we evaluated, for the different microcosms, the prevailing community assembly processes and related them to the subsequent invasion outcome.

\section{Stochastic processes determine invasion success in NOB guilds}

Resident communities, assembled with different dominating processes, were subject to invasion at different propagule pressures. We did not see a consistent correlation between the average distance from the resident community and invader relative abundance, hence our observations 
indicated that selection did not govern invasion outcome. When drift dominates invasion, incidence and relative abundance of the invader are expected to increase with propagule pressure. Based on this, we observed support for drift as governing process of invasion.

First, in batch microcosms, where the selection to drift ratio was lowest, we observed a clear effect of propagule pressure on invasion outcome (Table 1). Both frequency of invader establishment, as well as relative abundance of invader increased in response to higher concentrations of added invader cells, supporting drift as the process governing invasion in batch microcosms.

Second, in flow-through microcosms, successful establishment was only observed at high propagule pressure (Table 1). Failure to establish at lower propagule pressures could be a result of drift supported by the characteristics of a flow-through microcosm, where the actual propagule pressure in the system is lower than the theoretical propagule pressure because invader cells could easily flow through the system without attaching to the biofilm surface. Fewer invader cells are more affected by drift and the probability of extinction is increased, compared to larger populations.

Interestingly, drift explained invasion success also in resident communities where the relative importance of selection was high. This is somewhat unexpected, since in communities governed by selection, the competition caused by the fitness difference between invader and resident community members was expected to govern invasion success. One explanation could be that the high phylogenetic similarity of invader and resident community members reduced competition due to absence of large fitness differences. Our observations were made using natural communities where phylogenetic distances between community members and the invader are very low compared to many other invasion experiments with synthetic communities where phylogenetic distances are up to 10-fold higher $[11,46]$. However, when similarly low phylogenetic diversity and low phylogenetic distance between invader and resident were investigated, a similar conclusion was reached: propagule pressure increased invasion success and phylogenetic diversity had no effect on invasion success [21].

In conclusion, our results suggest that for functional guilds invaded by a guild member, where phylogenetic distance between resident and invader is typically low, stochastic processes govern invasion success, even when the relative importance of selection in the resident community is high. Our results also imply that predicting invasion of functional guilds by a member of the same guild from compositional information is nearly impossible, making the precise characterization of the composition of resident communities futile for this purpose. While regular measurements targeting the relative abundance of possible invader can be used to estimate the probability of establishment, the stochastic nature of drift does not allow predictions with high certainty. These observations need to be verified for functional guilds that have opportunities for larger ecological differences, to test if we can generalize our findings across any type of invasion in microbial communities.

Acknowledgements This work was funded by the Marie SkłodowskaCurie Actions of the European Union's Seventh Framework Programme FP7/2007-2013/ (MERMAID ITN) under REA grant agreement $n^{\circ} 607492$. In addition, we would like to thank Professor Eva Spieck from the University of Hamburg for donating the Nitrotoga HW29 culture and Chiara Ilgrande from Ghent University for guidance on culturing NOBs.

Author contributions $\mathrm{MK}, \mathrm{AD}$, and BFS designed the experiments. MK performed the experiments and carried out all molecular analyses; the data analysis was performed by MK supported by AD. All coauthors assisted in interpreting the results; MK initiated the manuscript writing, which was finalized with contributions from $\mathrm{AD}, \mathrm{H}-\mathrm{JA}$ and BFS.

\section{Compliance with ethical standards}

Conflict of interest The authors declare that they have no conflict of interest.

\section{References}

1. Mallon CA, Elsas JD van, Salles JF. Microbial invasions: the process, patterns, and mechanisms. Trends Microbiol. 2015a;23:719-29.

2. Amalfitano S, Coci M, Corno G, Luna GM. A microbial perspective on biological invasions in aquatic ecosystems. Hydrobiologia. 2014;746:13-22.

3. De Schryver P, Vadstein O. Ecological theory as a foundation to control pathogenic invasion in aquaculture. ISME J. 2014;8:2360-8.

4. Emery SM, Gross KL. Dominant species identity, not community evennes, regulates invasion in experimental grassland plant communities. Ecology. 2007;88:954-64.

5. Fargione JE, Tilman D. Diversity decreases invasion via both sampling and complementarity effects. Ecol Lett. 2005;8:604-11.

6. Mallon CA, Poly F, Le Roux X, Marring I, van Elsas JD, Salles JF. Resource pulses can alleviate the biodiversity-invasion relationship in soil microbial communities. Ecology. 2015b;96:915-26.

7. Elton CS. The ecology of invasions by animals and plants. Boston, MA: Springer US; 1958.

8. Darwin C. On the origin of species. New York, NY: D. Appleton and Co.; 1859.

9. Jiang L, Tan J, Pu Z. An experimental test of Darwin's naturalization hypothesis. Am Nat. 2010;175:415-23.

10. Procheş Ş, Wilson JRU, Richardson DM, Rejmánek M. Searching for phylogenetic pattern in biological invasions. Glob Ecol Biogeogr. 2008;17:5-10.

11. Tan J, Pu Z, Ryberg Wa, Jiang L. Resident-invader phylogenetic relatedness, not resident phylogenetic diversity, controls community invasibility. Am Nat. 2015;186:59-71.

12. Thuiller W, Gallien L, Boulangeat I, de Bello F, Münkemüller T, Roquet C, et al. Resolving Darwin's naturalization conundrum: A quest for evidence. Divers Distrib. 2010;16:461-75. 
13. van Elsas JD, Chiurazzi M, Mallon CA, Elhottova D, Kristufek V, Salles JF. Microbial diversity determines the invasion of soil by a bacterial pathogen. Proc Natl Acad Sci USA. 2012;109: 1159-64.

14. Bonanomi G, Capodilupo M, Incerti G, Gaglione SA, Scala F. Fungal diversity increases soil fungistasis and resistance to microbial invasion by a non resident species. Biol Control. 2014;72:38-45.

15. Dillon RJ, Vennard CT, Buckling A, Charnley AK. Diversity of locust gut bacteria protects against pathogen invasion. Ecol Lett. 2005;8:1291-8.

16. Shade A. Diversity is the question, not the answer. ISME J. 2017;11:1-6.

17. Kinnunen M, Dechesne A, Proctor C, Hammes F, Johnson D, Quintela-Baluja M, et al. A conceptual framework for invasion in microbial communities. ISME J. 2016;10:2773-5.

18. De Roy K, Marzorati M, Negroni A, Thas O, Balloi A, Fava F, et al. Environmental conditions and community evenness determine the outcome of biological invasion. Nat Commun. 2013;4:1383.

19. Daleo P, Alberti J, Iribarne O. Biological invasions and the neutral theory. Divers Distrib. 2009;15:547-53.

20. Acosta F, Zamor RM, Najar FZ, Roe BA, Hambright KD. Dynamics of an experimental microbial invasion. Proc Natl Acad Sci. 2015;112:11594-99.

21. Ketola T, Saarinen K, Lindström L. Propagule pressure increase and phylogenetic diversity decrease community's susceptibility to invasion. BMC Ecol. 2017;17:15.

22. Von Holle B, Simberloff D. Ecological resistence to biological invasion overwhelmed by propagule pressure. Ecology. 2005;86:3212-8.

23. Lockwood JL, Cassey P, Blackburn T. The role of propagule pressure in explaining species invasions. Trends Ecol Evol. 2005;20:223-28.

24. Simberloff D. The role of propagule pressure in invasion success. Annu Rev Ecol Evol Syst. 2009;40:81-102.

25. Chapelle E, Mendes R, Bakker PaH, Raaijmakers JM. Fungal invasion of the rhizosphere microbiome. ISME J. 2015;10:1-4.

26. Eisenhauer N, Schulz W, Scheu S, Jousset A. Niche dimensionality links biodiversity and invasibility of microbial communities. Funct Ecol. 2013;27:282-8.

27. Hüpeden J, Wegen S, Off S, Lücker S, Bedarf Y, Daims H, et al. Relative abundance of Nitrotoga spp. in a biofilter of a coldfreshwater aquaculture plant appears to be stimulated by slightly acidic pH. Appl Environ Microbiol. 2016;82:1838-45.

28. Rittmann BE, McCarty PL. Model of steady-state-biofilm kinetics. Biotechnol Bioeng. 1980;22:2343-57.

29. Terada A, Lackner S, Kristensen K, Smets BF. Inoculum effects on community composition and nitritation performance of autotrophic nitrifying biofilm reactors with counter-diffusion geometry. Environ Microbiol. 2010;12:2858-72.

30. Stoddard SF, Smith BJ, Hein R, Roller BRK, Schmidt TM. $r r n D B$ : improved tools for interpreting rRNA gene abundance in bacteria and archaea and a new foundation for future development. Nucleic Acids Res. 2015;43:D593-8.
31. Kinnunen M, Gülay A, Albrechtsen H-J, Dechesne A, Smets BF. Nitrotoga is selected over Nitrospira in newly assembled biofilm communities from a tap water source community at increased nitrite loading. Environ Microbiol. 2017;19:2785-93.

32. Lim J, Do H, Shin SG and Hwang S. Primer and probe sets for group-specific quantification of the genera Nitrosomonas and Nitrosospira using real-time PCR. Biotechnol. Bioeng., 2008;99: 1374-1383. https://doi.org/10.1002/bit.21715

33. Dionisi HM, Layton AC, Harms G, Gregory IR, Robinson KG, \& Sayler GS. Quantification of Nitrosomonas oligotropha-like ammonia-oxidizing bacteria and Nitrospira spp. from full-scale wastewater treatment plants by competitive PCR. Applied and environmental microbiology, 2002;68:245-253.

34. Yu Y, Lee C, Kim J, Hwang S. Group-specific primer and probe sets to detect methanogenic communities using quantitative realtime polymerase chain reaction. Biotechnol Bioeng. 2005; 89:670-9.

35. Callahan BJ, McMurdie PJ, Rosen MJ, Han AW, Johnson AJ A, \& Holmes SP. DADA2: high-resolution sample inference from Illumina amplicon data. Nature methods, 2016;13:581.

36. McMurdie PJ, Holmes S, Phyloseq: an R package for reproducible interactive analysis and graphics of microbiome census data. PLoS ONE. 2013;8:e61217.

37. Tsirogiannis C, Sandel B. PhyloMeasures: a package for computing phylogenetic biodiversity measures and their statistical moments. Ecography (Cop). 2016;39:709-14.

38. Nowka B, Daims H, Spieck E. Comparison of oxidation kinetics of nitrite-oxidizing bacteria: Nitrite availability as a key factor in niche differentiation. Appl Environ Microbiol. 2015;81:745-53.

39. Vellend M. Conceptual synthesis in community ecology. Q Rev Biol. 2010;85:183-206.

40. Evans S, Martiny JBH, Allison SD. Effects of dispersal and selection on stochastic assembly in microbial communities. ISME J. 2017;11:176-85.

41. Zha Y, Berga M, Comte J, Langenheder S. Effects of dispersal and initial diversity on the composition and functional performance of bacterial communities. PLoS ONE. 2016;11:e0155239.

42. Lücker S, Schwarz J, Gruber-Dorninger C, Spieck E, Wagner M, Daims H. Nitrotoga-like bacteria are previously unrecognized key nitrite oxidizers in full-scale wastewater treatment plants. ISME J. 2014;9:708-20.

43. Albers CN, Ellegaard-Jensen L, Hansen LH, Sørensen SR. Bioaugmentation of rapid sand filters by microbiome priming with a nitrifying consortium will optimize production of drinking water from groundwater. Water Res. 2018;129:1-10.

44. Nemergut DR, Schmidt SK, Fukami T, O’Neill SP, Bilinski TM, Stanish LF, et al. Patterns and processes of microbial community assembly. Microbiol Mol Biol Rev. 2013;77:342-56.

45. Gallien L, Carboni M, Münkemüller T. Identifying the signal of environmental filtering and competition in invasion patterns - a contest of approaches from community ecology. Methods Ecol Evol. 2014;5:1002-11.

46. Naughton HR, Alexandrou MA, Oakley TH, Cardinale BJ. Phylogenetic distance does not predict competition in green algal communities. Ecosphere. 2015;6:art116. 